\title{
CORRECTION
}

\section{Author Correction: Asperosaponin VI promotes angiogenesis and accelerates wound healing in rats via up-regulating HIF-1 $\alpha /$ VEGF signaling}

Cheng-gui Wang ${ }^{1,2}$, Yi-ting Lou ${ }^{1,2}$, Min-ji Tong ${ }^{1,2}$, Li-lian Zhang ${ }^{1,2}$, Zeng-jie Zhang ${ }^{1,2}$, Yong-zeng Feng ${ }^{1,2}$, Shi Li $^{1,2}$, Hua-zi Xu ${ }^{1,2}$ and Cong Mao ${ }^{1,2}$

Acta Pharmacologica Sinica (2022) 43:1617-1618; https://doi.org/10.1038/s41401-021-00777-3

Correction to: Acta Pharmacologica Sinica https://doi.org/10.1038/ aps.2017.161, published online 7 December 2017

The authors apologize that the images in Fig. 3B appeared incorrectly. We misassembled the tube formation images. The correct tube formation images in Fig. 3B are given. This correction does not affect the conclusions of the original article. We apologize again for any inconvenience caused.

The correct Fig. 3 is shown below.

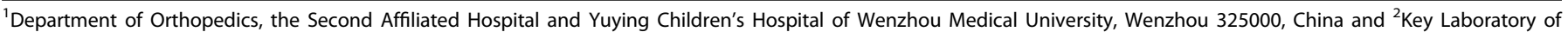
Orthopedics of Zhejiang Province, the Second Affiliated Hospital of Wenzhou Medical University, Wenzhou 325000, China

Correspondence: Hua-zi Xu (spinexu@163.com) or Cong Mao (maocong@wmu.edu.cn)

Published online: 15 September 2021 
A

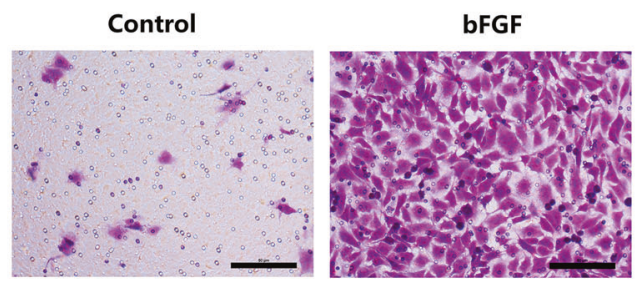

ASA VI $(\mu \mathrm{g} / \mathrm{ml})$
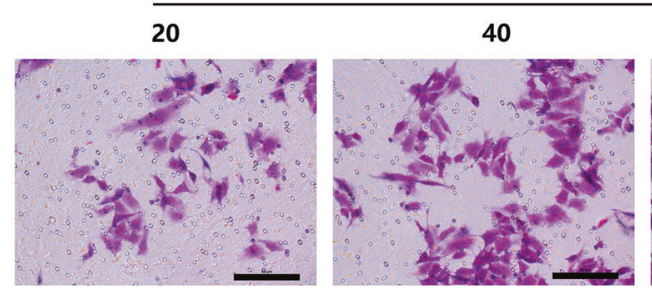

B
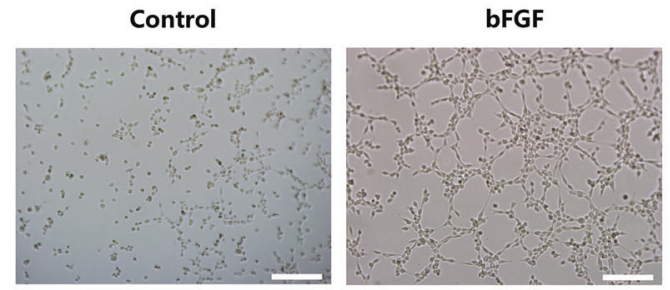

ASA VI $(\mu \mathrm{g} / \mathrm{ml})$
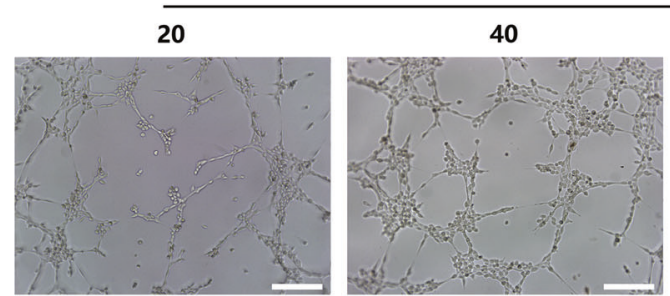

80

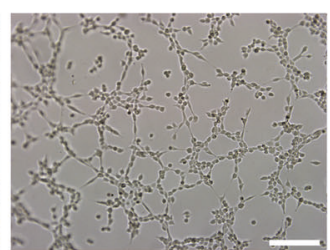

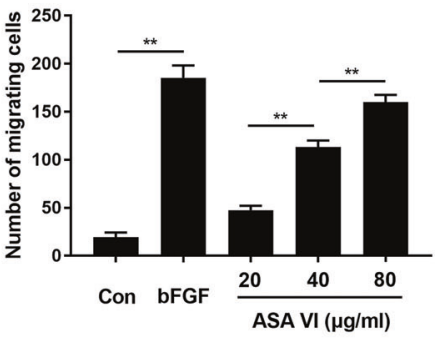

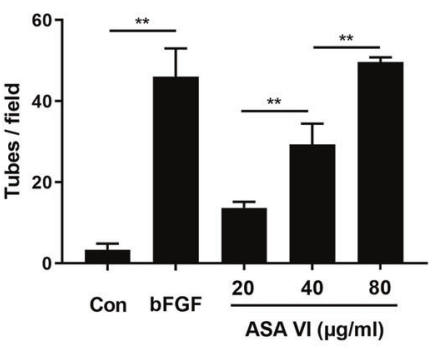

Fig. 3 ASA VI enhanced migration and tube formation of HUVECs. A Transwell migration assay results of HUVECs with different treatments. HUVECs were treated with PBS (control), $50 \mathrm{ng} / \mathrm{mL}$ bFGF and 20, 40, and $80 \mu \mathrm{g} / \mathrm{mL}$ ASA Vl in the lower chamber for $3 \mathrm{~h}$ incubation. The migration of HUVECs was enhanced after ASA VI treatment (scale bar: $50 \mu \mathrm{m}$ ). B In vitro tube formation results of HUVECs treated by ASA VI. The tube formation ability of HUVECs was improved after ASA VI treatment (scale bar: $200 \mu \mathrm{m}$ ). $n=3$ independent experiments. ${ }^{*} P<0.05$, ${ }^{* *} P<0.01$ 